\title{
INFLUENCIA DEL HUMUS DE LOMBRICULTURA EN EL CRECIMIENTO INICIAL DE CEDRO COLORADO EN PLANTACION A CAMPO ABIERTO Y COMPORTAMIENTO AL ATAQUE DE Hypshiphylla sp.
}

Américo Quevedo Guevara *

\section{RESUMEN}

El presente estudio trata de corregir limitantes de baja fertilidad de suelos degradados en Pucallpa y alta mortalidad por ataque de hypsiphylla sp. en las plantaciones de Cedro colorado a campo abierto con aplicación localizada de humus de lombriz en dosis de 2 y $4 \mathrm{lg}$ !planta, con un testigo (suelo) como patrón comparativo en parcelas experimentales con 4 bloques y 3 tratamientos, se orienta a vigorizar el plantón para aligerar su crecimiento y reducir el índice de mortalidad mediante el rebrote de nuevas yemas.

Los resultados obtenidos se sintetizan en: a) El crecimiento de cedro colorado con dosis de 2 y $4 \mathrm{Kg}$. de humus de lombriz, tuvo un desarrollo significativo en los 365 días que duró el estudio, manifestándose un incremento superior al $400 \%$ en los parámetros de altura y diámetro con respecto al testigo. b) El índice de mortalidad en Cedrela odorata se redujo a $10 \%$ en los tratamientos con $4 \mathrm{Kg}$. y $2 \mathrm{Kg}$. de humus de lombriz, por efectos del alto vigor de los plantones.

En ambos parámetros, crecimiento y mortalidad, se establece una diferencia estadística significativa en los tratamientos con $4 \mathrm{Kg}$. y $2 \mathrm{Kg}$. aun nivel de $0.05 \%$ con respecto al testigo; no existiendo tal diferencia entre ambos tratamiento con humus de lombriz.

Los niveles de materia orgánica presente en el humus de lombriz., manifiestan alto poder residual al año de su aplicación.

\section{INTRODUCCION}

La extracción comercial de especies forestales de alto valor como cedro colorado, muestra signos de agotamiento en los bosques amazónicos. Evidencias de esto son una saca más difícil y la reducción anual de volúmenes rollizos. Por otro lado, la intensiva extracción de estas especies no guarda relación con el

* Investigador IIAP. Centro Regional de Investigación IIAP-Ucayali. 
número de especies reforestadas logradas artificialmente, a pesar de los esfuerzos realizados por superar los problemas silviculturales que se presentan.

Entre estos problemas destacan el lento crecimiento en suelos de baja fertilidad y muy pobre contenido de MO y en ambientes ecológicos diferentes a su hábitat y los altos Índices de mortalidad por ataque de Hypsiphylla sp. Predator específico y endémico para Meliáceas en trópico húmedo.

La importancia de la MO como fuente de nutrientes para la planta es relevada por Zirena y Díaz (1983) y por Sánchez (1981), quienes sostienen que el mejoramiento de la fertilidad del suelo se logra con la aplicación de abonos orgánicos para mejorar su estructura y constituir fuente de nutrientes y reguladores del pH, Konova y Oblitas (1983), y Dyson (1981) destacan la gran importancia de la MO en el suelo por el aporte gradual de nutrientes luego de la mineralización y humificación, por favorecer el incremento de la capacidad de intercambio catiónico, por la retención de humedad y por la agregación del suelo.

Se considera al humus de lombriz como el abono orgánico de mayor relevancia para cultivos por las bondades físicas, químicas y biológicas. Además mejoran considerablemente la estructura del suelo, regula el $\mathrm{pH}$, aporta adecuados niveles de micronutrientes difícilmente disponibles en el suelo y adecuados niveles de $\mathrm{N}, \mathrm{P}, \mathrm{K}, \mathrm{Ca}$ y $\mathrm{Mg}$. Igualmente eleva la población de microorganismos, los que incrementan los niveles de nutrición y fomentan la producción de hormonas de crecimiento como las auxinas y giberelinas $(1,6)$. Estas hormonas actúan como impulsores del creciento en cultivos anuales $(2,3)$. En la Amazonía esta premisa para especies forestales se inicia con el presente estudio para cedro colorado; sin embargo, es importante resaltar los trabajos de Vega (1976 y 1979) en Suriname en Cedrela angustifolia. Este autor obtuvo exitosos resultados mediante la acumulación de materia orgánica del bosque primario en "camellones". Este sustrato contiene considerables porcentajes de humus natural, producto de la actividad microbiana del suelo.

La búsqueda de resultados que permitan la reforestación de cedro en condiciones de campo abierto y en suelos degradados motivó este estudio. Para el experimento se usó humus de lombriz durante el transplante como sustrato sustitutorio del suelo en forma localizada estratificada. Esta es una metodología que desarrolla el IIAP (Instituto de Investigaciones de la Amazonía Peruana). Este tratamiento debe, además, aligerar el crecimiento longitudinal y reducir los índices de mortalidad mediante el rebrote de nuevas yemas apicales luego del ataque de Hypsipylla sp. La base de esto es el vigor fisiológico transmitido por las bondades tísicas, químicas, y biológicas del humus de lombriz que permite al cedro colorado superar eficientemente condiciones adversas de suelo y de ataque. 


\section{MATERIALES Y MÉTODOS}

El estudio se efectuó en la Estación Experimental del Centro Regional de Investigación del IIAP-Ucayali; ubicada en el Km. 12 de la Carretera Federico Basadre en la ciudad de Pucallpa. El suelo del estudio corresponde probablemente a un ultisol, por sus características de acidez y baja fertilidad (cuadro 1).

Cuadro 1

ANÁLISIS DE SUELO

ANALISIS MECAN1CO $\quad \mathrm{pH}$ CO3CA $\mathrm{MO} \quad \mathrm{N} \quad \mathrm{P}$ CAMBIABLES m e/100 g SAT Arena Limo Arcilla Textura 1:1 \% $\% \quad \% \quad$ ppm $\mathrm{Ca}+\mathrm{Ma} \mathrm{Al}+\mathrm{H} \quad \mathrm{Al} \mathrm{CIC}$ A $\%$

$\% \quad \% \quad \%$

$\begin{array}{llllllllllllll}32 & 40 & 25 & \text { Franco } & 4.5 & 0.0 & 1.7 & 0.076 & 2.6 & 16 & 2.4 & 1.6 & 4.0 & 40.0\end{array}$

Fuente: Laboratorio de análisis de suelo de la U.N.A.S. - Tingo María (1991)

El estudio se basó en la aplicación de 0,2 y $4 \mathrm{~kg}$. de humus de lombriz por planta. Las características químicas del humus de lombriz se las presenta en el cuadro 2 .

Cuadro 2

CARACTERÍSTICAS QUIMICAS DEL HUMUS DE LOMBRIZ

\begin{tabular}{lllllll}
$\mathrm{pH}$ & $\begin{array}{l}\mathrm{MO} \\
\%\end{array}$ & $\begin{array}{l}\mathrm{N} \\
\%\end{array}$ & $\begin{array}{l}\mathrm{P} \\
\%\end{array}$ & $\begin{array}{l}\mathrm{K} \\
\%\end{array}$ & $\begin{array}{l}\text { C.I.O } \\
\text { mmhos/ }\end{array}$ & $\begin{array}{l}\mathrm{MO} \\
\mathrm{u} / \mathrm{g}\end{array}$ \\
\hline 6.7 & 50.43 & 1.2 & 0.95 & 0.47 & 2.1 & $33^{\prime} 360,000$
\end{tabular}

Fuente: Elaborado en el Laboratorio de suelos y plantas - UNA - La Molina; publicado por el IIAP-Ucayali

La plantación se realizó a campo abierto en setiembre de 1992 y con un distanciamiento de 1 x $1 \mathrm{~m}$ usando plantones de cedro con un $100 \%$ de ataques de Hypsiphylla sp. Los plantones se arreglaron en bloques. Los plantones para el 
tratamiento de $\mathrm{O} \mathrm{kg} /$ planta tuvieron un promedio de $0.58 \mathrm{~cm}$ de diámetro y 34.0 $\mathrm{cm}$ de altura. Los plantones del tratamiento $2 \mathrm{~kg} /$ planta tuvieron promedio de $0.56 \mathrm{~cm}$ de diámetro y $29.3 \mathrm{~cm}$ de altura. Los plantones para el tratamiento de 4 $\mathrm{kg} /$ planta de humus tuvieron un promedio de $0.56 \mathrm{~cm}$ de diámetro y $28.6 \mathrm{~cm}$ de altura.

Para la plantación se hizo hoyos de 20x $20 \mathrm{~cm}$ y $45 \mathrm{~cm}$ de profundidad. Las diferentes dosis de humus se aplicó en estratos de $15 \mathrm{~cm}$ intercalados con suelo. En el caso del tratamiento sin humus se removió el suelo a la misma profundidad.

Se usó un diseño de bloques completamente el azar con 4 repeticiones. Se evaluó crecimiento y mortalidad. El crecimiento se sustentó en la evaluación de la altura y diámetro. La mortalidad se evaluó por el número de plantas muertas.

En la evaluación se hizo el registro en 24 oportunidades, con intervalos de 15 días.

\section{RESULTADOS}

Hasta los 365 días de crecimiento la aplicación de 2 y 4 kg de humus de lombriz por planta, elevó significativamente $(\mathrm{p} \leq 0.05)$ el incremento de diámetro y de altura de las plantas de cedro (gráfico 1). No hubo, sin embargo, diferencias estadísticas entre los tratamientos de 2 y $4 \mathrm{~kg}$ de humus por planta.

El porcentaje de mortalidad se redujo significativamente $(\mathrm{p} \leq 0.05)$ en los tratamientos con abono comparados al porcentaje de mortalidad de las plantas sin humus. Entre los tratamientos con 2 y $4 \mathrm{~kg} /$ planta los porcentajes de mortalidad son similares.

Por otro lado el porcentaje de rebrote de las plantas tratadas con humus, se incrementó significativamente $(\mathrm{p} \leq 0.05)$ gráfico 2 comparados al testigo.

- Cedro colorado, luego del ataque del insecto en condiciones de vigor fisiológico, recupera la forma cilíndrica del tallo.

- El contenido de materia orgánica del humus de lombriz disminuye en un $30 \%$ al año de su aplicación, lo que le confiere alto poder residual nutritivo.

- $\quad$ El pH inicial de 6.7 del abono, se mantiene invariable al año de su aplicación en el sitio localizado en plena actividad fisiológica de cedro colorado.

\section{DISCUSION}

\section{CRECIMIENTO}

El valor promedio de $129 \mathrm{~cm}$ de crecimiento en altura y $2.66 \mathrm{~cm}$ en diámetro obtenido en 12 meses de evaluación para 2 y $4 \mathrm{~kg}$ de humus por planta 
es ligeramente superior a los 120 y $2.5 \mathrm{~cm}$ reportados por Vega (1979) a 22 meses en similar método de plantación.

Este crecimiento favorable de cedro colorado con el humus, permite esperar mejores efectos en el tiempo, si consideramos que las condiciones edáficas del sitio utilizado por Vega (1979) son las de un terreno recientemente deforestado. En cambio, las condiciones del sitio de estudio, corresponden a características típicas de suelos degradados de predios agropecuarios en ejes de carretera sometidos a actividades agrícolas y pecuarias intensivas no acorde a su capacidad de uso.

El crecimiento inicial logrado puede deducirse como efecto de las propiedades físicas, químicas y biológicas del humus de lombriz, favorecidas con adecuada textura, capacidad retentiva de humedad, alto contenido de $\mathrm{MO}, \mathrm{pH}$, neutro, adecuados niveles de macro y micro nutrientes, elevada capacidad de cambio, así como abundantes microorganismos cuya actividad en el sustrato permite a la planta disponer continuamente de elementos esenciales en el crecimiento como las fitohormonas.

El hecho que no haya diferencia estadística de crecimiento de cedro colorado entre 2 y $4 \mathrm{~kg}$ de humus por planta sugiere la dosis de $2 \mathrm{~kg} /$ plantón como económica y fisiológicamente adecuada para que la especie logre un crecimiento inicial acelerado y vigoroso en su primer año de instalación en el sitio definitivo. Este efecto influye en la reducción de costos por labores de mantenimiento y reposición por mortalidad, y confiere a la especie viabilidad técnico-económica para su propagación en condiciones de campo abierto.

\section{MORTALIDAD}

La reducción a $10 \%$ de mortalidad logrado para cedro colorado en el presente experimento es superior al $45 \%$ reportado por Vega (1979) en condiciones similares en un período de 0 a 2 años, edad de máxima incidencia de ataque de Hypsiphyla sp. Asimismo, la resistencia y recuperación fisiológica de la especie en el estudio es superior a lo registrado en las plantaciones su Suriname (Vega 1979).

Es importante resaltar que el índice de $10 \%$ de mortalidad aconteció en los dos primeros meses de siembra (octubre y noviembre) en el sitio definitivo. Este período correspondió a época de alta 1ecipitación en la Región Ucayali, condiciones favorables para la producción de Hypsiphylla sp. y condiciones de estadio incipiente de vigor de cedro colorado, en tanto la planta se encontraba en una etapa de recuperación de los daños ocasionados por el insecto en vivero.

La reducción de los índices de mortalidad de cedro colorado, cuando es tratado con humus de lombriz en su crecimiento inicial, sugiere que no es menester distraer esfuerzos y recursos en la persecución de Hypsiphylla sp., sino 
más bien; dotar a la planta de un sustrato rico en nutrientes, microorganismos y adecuado $\mathrm{pH}$, condiciones que exige la especie para responder al daño del insecto logrando mayores incrementos longitudinales de los rebrotes luego del ataque.

Esta característica fisiológica es una respuesta a los niveles adecuados de nutrientes y sustancias hormonales. Esto manifiesta por el inmediato rebrote de nuevas yemas apicales luego del ataque, las que deben seleccionarse, dejando únicamente aquella que presente el menor ángulo al tallo para favorecer el empalme recto.

\section{CONCLUSIONES}

La velocidad de crecimiento en el diámetro y altura en plantones de cedro con humus de lombriz es acelerada en grado significativo, registrándose incrementos superiores al $400 \%$ en ambos parámetros en el crecimiento inicial.

Es posible plantar cedro en campo abierto, enriqueciendo el sustrato con humus de lombriz en suelos típicos de predios agropecuarios. Esto debido a las adecuadas propiedades físicas, químicas y biológicas del humus.

Se puede reducir la mortalidad del cedro por Hypsiphylla sp. a $10 \%$ por efecto del vigor en el plantón como consecuencia de la presencia de nutrimentos y la actividad microbiana disponible en el humus de lombriz. Esto reduce los costos de reposición.

Los mismos resultados de crecimiento se obtienen con dosis de humus de 2 y $4 \mathrm{~kg}$; por lo que la dosis económica sería de $2 \mathrm{~kg} /$ plantón, en forma localizada.

La inclusión de humus de lombriz con aplicación localizado estratificada, como abono orgánico en plantaciones a campo abierto, no eleva significativamente los costos por su bajo valor en el mercado y por la factibilidad de ser producido por pequeños productores que dispongan de un hato ganadero de 1-30 cabezas o granjas avícolas o de ovinos.

Los plantones de cedro colorado con 2 y $4 \mathrm{~kg}$ de humus por planta recuperaron su vigor y forma, reiniciando un rápido enraizamiento y crecimiento, y notándose una mejora sustancial en el aspecto fitosanitario.

La velocidad del crecimiento inicial del cedro colorado con humus de lombriz reduce la periodicidad de labores de limpieza de malezas a dos durante el año, bajando significativamente los costos de mantenimiento.

Los nuevos fustes emergentes de los rebrotes, luego de un ataque de Hypsiphylla sp en plantones tratados con humus de lombriz, muestran un acelerado crecimiento longitudinal por segmentos; el que se estabiliza para dar lugar a un empalme satisfactorio de su diámetro al del fuste original dejando mostrar únicamente un ligero anillo como efecto del ataque, siendo necesario para ello eliminar la parte perforada por el insecto para evitar la invasión de hormigas y termites. 


\section{BIBLIOGRAFIA}

BANCO AGRARIO. 1987. "Manual de instrucciones para lombricultura”. Dpto. de Divulgación Técnica. Lima-Perú. 47 p.

UAP-CORDEU. 1988. "La Lombricultura integrada a la actividad agrosilvopecuaria”. Pucallpa, Perú. 30 p.

IIAP-CORDEU. 1989. "Lombricultura y biotecnología”. Pucallpa, Perú.30 p. DYSON, W.G. 1981. "Fertilización de plantaciones forestales en la reserva forestal de La Yeguada". Turrialba, Costa Rica. 70 p.

KONOVA, y OBLITAS. 1982. Materia orgánica del suelo, su naturaleza, propiedades y métodos de investigación. Barcelona- España. $365 \mathrm{P}$.

SABAC-CHILE. 1980. "Centro de Desarrollo de la Lombricultura”. Santiago de Chile, Chile. 28 p.

SANCHEZ, P. 1981. Suelos del trópico, características y manejo. IICA. San José, Costa Rica. IICA. 634 p.

VEGA, L. 1976. "Plantaciones en Suriname”. Turrialha, Costa Rica, 39p.

VEGA, L. 1979. “La Silvicultura de Cedrela en Campo VI11”. Suriname. 35 p.

ZIRENA, D. y DIAZ N. 1983. "Fertilizantes, características y su utilización como bioabono". Cajamarca, Perú, 81 p. 


\section{Grafico 1}

INCRMENTO EN ALTURA Y DIAMETRO DE 120 PLANTAS DE CREDO A LOS 365 DIAS DE CRECIMIENTO COMO EFECTO DE LA APLICACIÓN DE DIFERENTES CANTIDADES DE HUMUS DE LOMBRIZ POR PLANTA. PROMEDIO DE 4 REPETICIONES
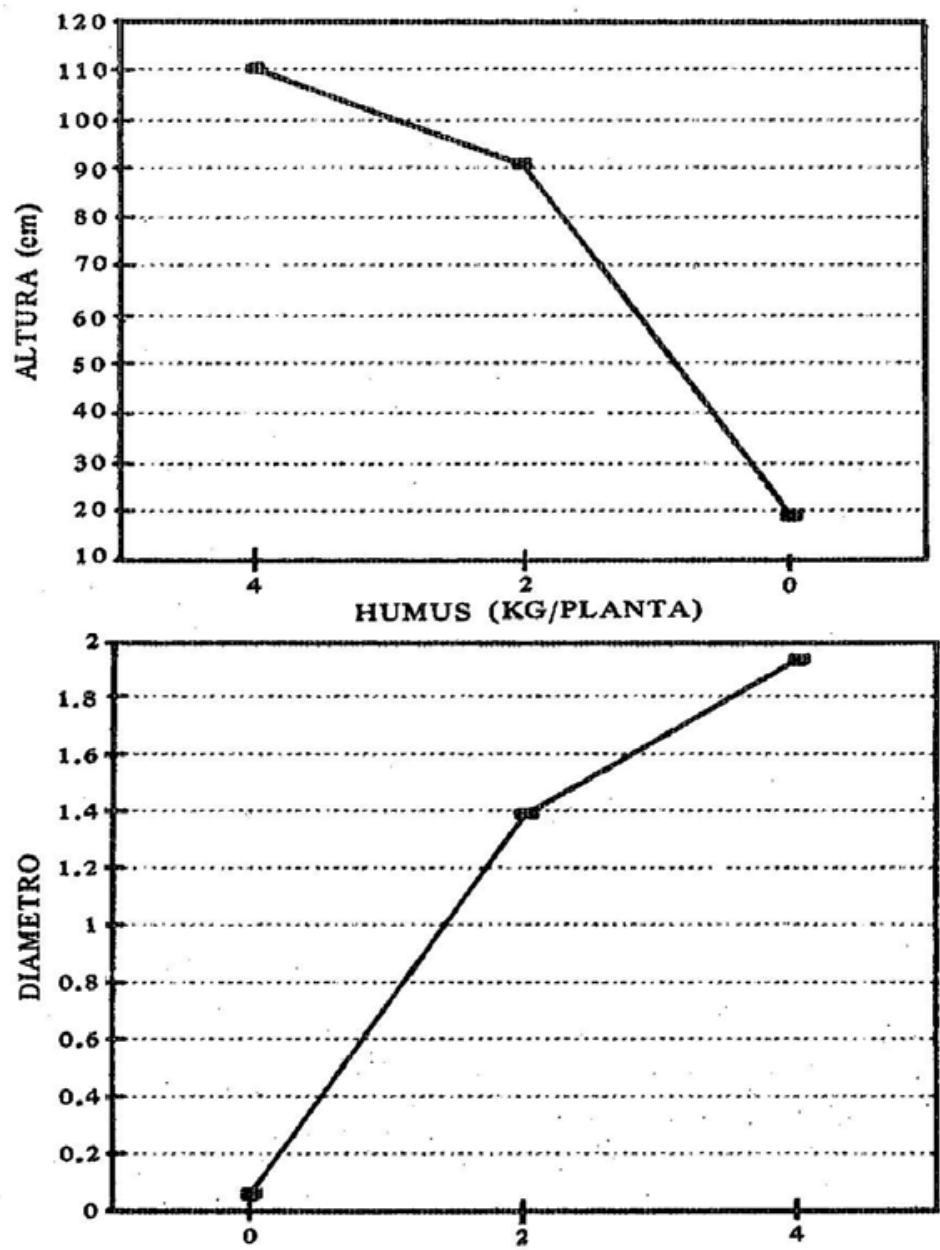


\section{Grafico 2}

PORCENTAJE DE MORTALIDAD Y REBROTE DE 108 PLANTAS DE CREDO A LOS 365 DIAS DE CRECIMIENTO COMO EFECTO DE LA APLICACIÓN DE DIFERENTES CANTIDADES DE HUMUS DE LOMBRIZ.
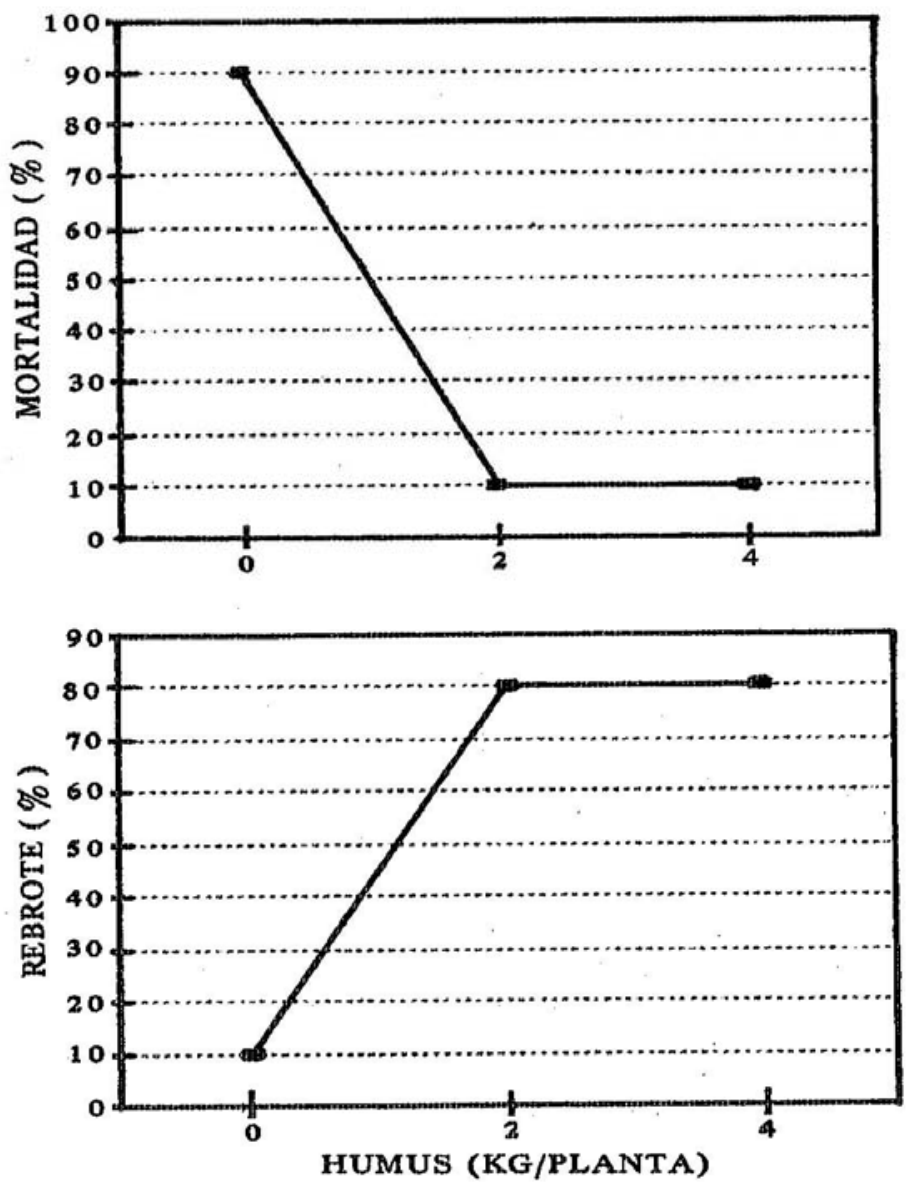